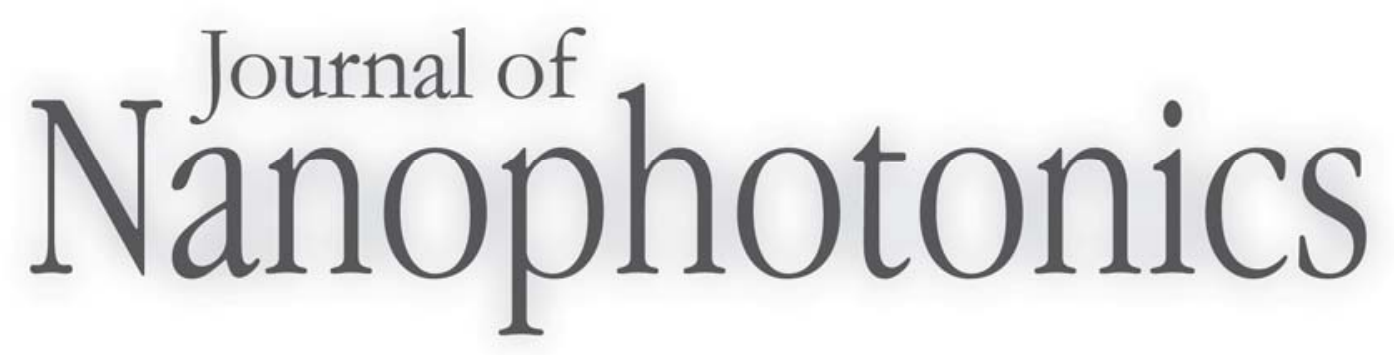

SPIEDigitalLibrary.org/jnp

\title{
Multiple light scattering and optomechanical forces
}

David L. Andrews

David S. Bradshaw

Luciana C. Dávila Romero 


\title{
Multiple light scattering and optomechanical forces
}

\author{
David L. Andrews, David S. Bradshaw, and Luciana C. Dávila Romero \\ University of East Anglia, Nanostructures and Photomolecular Systems, School of Chemistry, \\ Norwich NR4 7TJ, United Kingdom \\ david.andrews@physics.org
}

\begin{abstract}
When off-resonant light travels through a transparent medium, light scattering is the primary optical process to occur. Multiple-particle events are relatively rare in optically dilute systems: scattering generally takes place at individual atomic or molecular centers. Several well-known phenomena result from such single-center interactions, including Rayleigh and Raman scattering, and the optomechanical forces responsible for optical tweezers. Other, less familiar effects may arise in circumstances where throughput radiation is able to simultaneously engage with two or more scattering sites in close, nanoscale, proximity. Exhibiting the distinctive near-field electromagnetic character, inter-particle interactions such as optical binding and a variety of inelastic bimolecular processes can then occur. Although the theory for each two-center process is well established, the connectivity of their mechanisms has not received sufficient attention. To address this deficiency, and to consider the issues that ensue, it is expedient to represent the various forms of multi-particle light scattering in terms of transitions between different radiation states. The corresponding quantum amplitudes, registering the evolution of photon trajectories through the material system, can be calculated using the tools of quantum electrodynamics. Each of the potential outcomes for multi-particle scattering generates a set of amplitudes corresponding to different orderings of the constituent photon-matter interactions. Performing the necessary sums over quantum pathways between radiation states is expedited by a state-sequence development, this formalism also enabling the identification of intermediate states held in common by different paths. The results reveal the origin and consequences of linear momentum conservation, and they also offer new insights into the behavior of light between closely neighboring scattering events.
\end{abstract}

Keywords: Light scattering, Rayleigh and Raman scattering, dispersion forces, optical forces, optical tweezers, nonlinear optics, quantum electrodynamics.

\section{INTRODUCTION}

The scattering of light is a phenomenon that creates our visual world. Most of what we see directly results from diffuse surface reflection - notwithstanding that its color is primarily due to the depletion of other, absorbed wavelengths. Coming from the sky, most of our daylight is itself first scattered in the atmosphere, without significant absorption occurring. To determine a satisfactory answer to the ancient, yet surprisingly complex, question 'Why is the sky blue?' proved extraordinarily difficult until the arrival of Lord Rayleigh's scattering law [1]. Although this groundbreaking theory did not quite complete an explanation for the perceived color of daylight, it was the first major step towards our present understanding $[2,3]$, and it provided a basis for the subsequent development of atmospheric optics. A definitive and thorough review on the scattering of light in such a context, written in terms of modern classical electrodynamics, can be found in the excellent and widely used text book co-authored by Craig Bohren [4].

During the twentieth century, the advent of first quantum mechanics and then quantum electrodynamics (QED) led to a parallel development of theory for other optical phenomena, 
where the photonic character of visible radiation is more directly evident. In fact, quantization of the electromagnetic field is the only legitimate basis for utilizing the now pervasive concept of the photon. Comprehensive in its sweep, the quantized field formulation of QED has proved highly successful and remarkably accurate in describing the interactions of electromagnetic radiation with matter. As such, it now affords a consistent theoretical basis for processes that extend well beyond, and also subsume, those entailing the scattering of light. Although the results for light scattering delivered by such a theory are ultimately the same as those predicted by a classical construction - and necessarily so, since those agree with experiment - the case for entertaining the quantized field representation in this connection can stand on its own merits. First, it is a theory that unveils connections between processes which could not become apparent in the classical framework; moreover, QED offers a rigorous basis for considering more exotic phenomena. This article aims to demonstrate these aspects in the course of a development that connects familiar scattering processes occurring in the Rayleigh regime through to new results on multi-particle scattering and optically induced dispersion forces, at the heart of what has attracted the soubriquet 'optical matter' - see for example Refs. 5-7.

In the following, the familiar single-center light-matter interactions of Rayleigh and Raman scattering are first addressed in Sec. 2. Attention then focuses on other, less wellknown effects which may arise in circumstances where throughput electromagnetic radiation is able to simultaneously engage with two scattering sites, such as atoms or molecules in nanoscale proximity. In these situations, to entertain pairwise scattering of the radiation one must also account for inter-molecular electromagnetic couplings. Three of these higher-order processes are assessed in detail: optical binding (Sec. 3.1), then two-center Rayleigh and Raman scattering (Secs. 3.2 and 3.3). Section 4 addresses multi-center scattering, and some of the key physical implications of the results are drawn out in a concluding Discussion.

\section{SINGLE-CENTER SCATTERING}

At the outset, it may be useful to highlight certain respects in which a quantum radiation representation departs from the familiar classical picture of elastic (or inelastic) scattering. In essence, the QED approach differs from classical theory in that the full system is quantized, i.e. both the matter and radiation are treated quantum mechanically. The photon that emerges from any scattering interaction is in no meaningful sense physically identifiable with its input antecedent; both input and output photons enumerate the units of change in distinct radiation mode occupancies - the sole exception being forward Rayleigh scattering, where only one optical mode is involved. Moreover, in any scattering process, two distinct time-orderings of the input photon annihilation and emergent photon creation events are necessarily entertained, their quantum amplitudes being summed. Mathematically this reflects the fact that, in each individual multipolar interaction of any order, only one photon event (creation or annihilation) can occur. Thus there are two virtual states that, whilst each of immeasurably short duration, in a certain sense represent intermediates within the scattering process: in one case the input photon annihilation precedes the creation event, so that neither is present in the intermediate state; in the other, both photons are simultaneously present. Physically, the addition of amplitudes for these two quantum pathways can be understood as indicating the impossibility of precise photon localization in space or time, a manifestation of the photon's intrinsically quantum mechanical character, as sketched in Fig. 1. Whilst the virtual

\footnotetext{
* In this article we use the term particle to denote a nanoscale scattering center. In connection with Rayleigh or Raman scattering, these centers will be molecules.
} 
states need not conserve energy - hence their severely limited lifetime, indicated by $\Delta t$ in Fig. 1 - completion of the scattering process restores energy conservation.

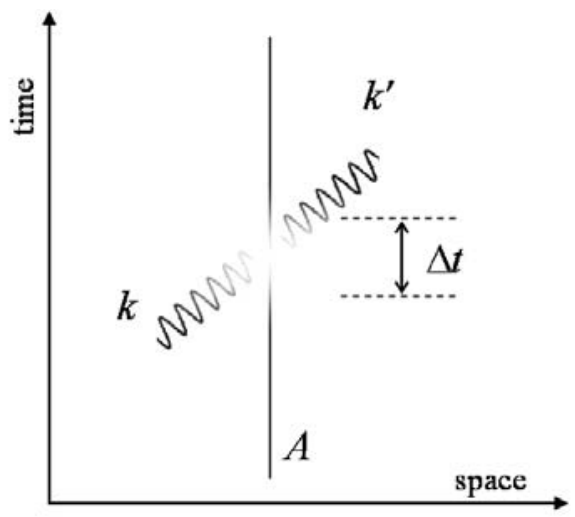

Fig. 1. Rayleigh scattering schematically depicted with respect to a time-space frame. The scatterer $A$ is represented by a vertical straight line (its kinetic energy considered negligible). The input $k$ and output $k^{\prime}$ radiation are represented by sinusoidal lines. The central fade-out represents a region where the radiation and scatterer states manifest quantum uncertainty.

In a complete quantum electrodynamical description, the non-relativistic Hamiltonian energy of a system, comprising molecules labeled $\xi$, is promoted to operator status to produce an operator, $H$, which in multipolar form [8] is exactly expressible as;

$$
H=\sum_{\xi} H_{\mathrm{mol}}(\xi)+\sum_{\xi} H_{\mathrm{int}}(\xi)+H_{\mathrm{rad}}
$$

where $H_{\text {mol }}$ is the molecular Hamiltonian, $H_{\text {rad }}$ is the radiation Hamiltonian and $H_{\text {int }}$ is the Hamiltonian representing the interaction of the radiation field with each molecule. On inspection of equation (1) it is immediately apparent that, in contrast to a classical description, pairwise coupling is not mediated by instantaneous coupling interactions - notice the absence of any terms with $\xi \neq \xi^{\prime}$ - but by the quantum field, whose photons exhibit retardation associated with their finite speed of propagation [9]; note that this is not the case if the minimal-coupling quantum formalism is employed. In Dirac notation the eigenstates, $|\Psi\rangle$, of a basis Hamiltonian given by equation (1) excluding $H_{\text {int }}$, form a composite set expressible in the form $|\Psi\rangle=\left|\operatorname{mol}_{n}\right\rangle\left|\operatorname{rad}_{n}\right\rangle \equiv\left|\operatorname{mol}_{n} ; \operatorname{rad}_{n}\right\rangle$, in which $\left|\operatorname{mol}_{n}\right\rangle$ defines the status of all molecules, comprising a product of state vectors for each molecule $\xi$, and in which $\left|\operatorname{rad}_{\mathrm{n}}\right\rangle$ is the radiation state. In the electric-dipole approximation, $H_{\text {int }}(\xi)$ is given by;

$$
H_{\mathrm{int}}(\xi)=-\varepsilon_{0}^{-1} \sum_{\xi} \mu(\xi) \cdot \mathbf{d}^{\perp}\left(\mathbf{R}_{\xi}\right),
$$

where the electric-dipole moment operator, $\mu(\xi)$, operates on matter (molecular) states, $\left|\mathrm{mol}_{\mathrm{n}}\right\rangle$, and the transverse electric displacement field operator, $\mathbf{d}^{\perp}\left(\mathbf{R}_{\xi}\right)$, operates on $\left|\operatorname{rad}_{\mathrm{n}}\right\rangle$; 
$\mathbf{R}_{\xi}$ is the position vector of molecule $\xi$. In explicit terms, $\mathbf{d}^{\perp}\left(\mathbf{R}_{\xi}\right)$ involves a summation over all wave-vectors, $\mathbf{k}$, and polarizations, $\lambda$, usually written as the following mode expansion;

$$
\mathbf{d}^{\perp}\left(\mathbf{R}_{\xi}\right)=\mathrm{i} \sum_{\mathbf{k}, \lambda}\left(\frac{\hbar c k \varepsilon_{0}}{2 V}\right)^{\frac{1}{2}}\left\{\mathbf{e}^{(\lambda)}(\mathbf{k}) a^{(\lambda)}(\mathbf{k}) e^{i\left(\mathbf{k} \cdot \mathbf{R}_{\xi}\right)}-\overline{\mathbf{e}}^{(\lambda)}(\mathbf{k}) a^{\dagger(\lambda)}(\mathbf{k}) e^{-\mathrm{i}\left(\mathbf{k} \cdot \mathbf{R}_{\xi}\right)}\right\}
$$

where $\mathbf{e}^{(\lambda)}(\mathbf{k})$ is the polarization unit vector $\left(\overline{\mathbf{e}}^{(\lambda)}(\mathbf{k})\right.$ being its complex conjugate), $V$ is an arbitrary quantization volume and $a^{(\lambda)}(\mathbf{k}), a^{\dagger(\lambda)}(\mathbf{k})$ are respectively the photon annihilation and creation operators for a mode $(\mathbf{k}, \lambda)$. The latter operators act on the radiation states through the general relations:

$$
\begin{gathered}
a^{(\lambda)}(\mathbf{k})|m(\mathbf{k}, \lambda)\rangle=\sqrt{m}|m-1(\mathbf{k}, \lambda)\rangle, \\
a^{\dagger(\lambda)}(\mathbf{k})|m(\mathbf{k}, \lambda)\rangle=\sqrt{m+1}|m+1(\mathbf{k}, \lambda)\rangle .
\end{gathered}
$$

The appearance in $H_{\text {int }}$ of the operators of (4), through (3), signifies its role in photon annihilation and creation.

\subsection{Rayleigh scattering}

In Rayleigh scattering - first taking the non-forward case where the scattered light undergoes a deflection from the incident direction - the initial state $|i\rangle$ may be written as $\left|E_{0}^{A} ; n(\mathbf{k}, \lambda) ; 0\left(\mathbf{k}^{\prime}, \lambda^{\prime}\right)\right\rangle$ and the final state $|f\rangle$ as $\left|E_{0}^{A} ; n-1(\mathbf{k}, \lambda) ; 1\left(\mathbf{k}^{\prime}, \lambda^{\prime}\right)\right\rangle$, using $A$ to represent the scatterer. Here, the subscript 0 denotes the ground energy level, and two radiation states are also specified. Single-centre scattering thus invokes both annihilation and creation operators - physically denoting photon annihilation and creation. The sequence in which the two operators are applied results in two routes from the initial to the final state, as illustrated by the state-sequence diagram of Fig. 2. Physically, these two sequences are interpreted as: ( $a$ ) the annihilation of a photon at $A$, followed by a subsequent photon creation; (b) vice-versa.

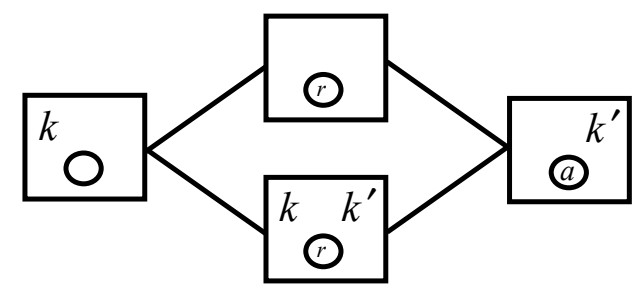

Fig. 2. State-sequence diagram for single-center scattering: a photon from mode $k$ is annihilated and one of mode $k^{\prime}$ is created. The initial state is on the left, the final state on the right; the two states in the middle are alternative intermediates. Here, an unfilled circle represents the ground state, and $r$ is an intermediate state; $a$ denotes an excited state in Raman scattering, but the ground state for elastic scattering. 
With two matter-photon interactions and $H_{\text {int }}$ acting as a perturbation, the quantum amplitude or matrix element, $M^{f i}$, for Rayleigh (elastic) scattering is calculated from the second term of an expansion in time-dependent perturbation theory;

$$
M^{f i}=\frac{\left\langle f\left|H_{\mathrm{int}}\right| r_{l}\right\rangle\left\langle r_{l}\left|H_{\mathrm{int}}\right| i\right\rangle}{\left(E_{i}-E_{r_{l}}\right)}+\frac{\left\langle f\left|H_{\mathrm{int}}\right| r_{u}\right\rangle\left\langle r_{u}\left|H_{\mathrm{int}}\right| i\right\rangle}{\left(E_{i}-E_{r_{u}}\right)},
$$

where the first and second terms correspond to the lower and upper pathways of Fig. 2, respectively. In the numerator expressions of the two terms in (5) one can identify the intermediate states $\left|r_{u}\right\rangle \equiv\left|E_{r}^{A} ;(n-1)(\mathbf{k}, \lambda) ; 0\left(\mathbf{k}^{\prime}, \lambda^{\prime}\right)\right\rangle$ and $\left|r_{l}\right\rangle \equiv\left|E_{r}^{A} ; n(\mathbf{k}, \lambda) ; 1\left(\mathbf{k}^{\prime}, \lambda^{\prime}\right)\right\rangle$; in the denominators, $E$ denotes the energy of each system state as signified by its subscript, such that $E_{i}=E_{0}+\hbar c k, \quad E_{r_{u}}=E_{r}, \quad E_{r_{l}}=E_{r}+2 \hbar c k$ and $E_{f}=E_{0}+\hbar c k$ (since $|\mathbf{k}|=\left|\mathbf{k}^{\prime}\right|$ for Rayleigh scattering). Inserting equation (2) into (5), the quantum amplitude for Rayleigh scattering, is determined: [10]

$$
M^{f i}=-\left(\frac{n^{\frac{1}{2}} \hbar c k}{2 \varepsilon_{o} V}\right) e_{i}^{(\lambda)}(\mathbf{k}) \bar{e}_{j}^{\left(\lambda^{\prime}\right)}\left(\mathbf{k}^{\prime}\right) \alpha_{i j}^{00(A)}(-k ; k) .
$$

Here, the implied summation convention for repeated Cartesian tensor indices is employed, and $\alpha_{i j}$ is the frequency-dependent polarizability tensor, given generally by:

$$
\alpha_{i j}^{m n(\xi)}\left( \pm k_{2} ; \pm k_{1}\right)=\sum_{s}\left\{\frac{\mu_{i}^{m s(\xi)} \mu_{j}^{s n(\xi)}}{E_{s n}^{(\xi)} \mp \hbar c k_{2}}+\frac{\mu_{j}^{m s(\xi)} \mu_{i}^{s n(\xi)}}{E_{s n}^{(\xi)} \mp \hbar c k_{1}}\right\},
$$

where the shorthand notation $\boldsymbol{\mu}^{x y}=\langle x|\boldsymbol{\mu}| y\rangle$ and $E_{x y}=E_{x}-E_{y}$ is employed. Again, within equation (7), the first term corresponds to the lower pathway of Fig. 2 and the second term to the upper pathway.

\subsection{Raman scattering}

Similar results can be drawn for the inelastic Raman counterpart, i.e. $|\mathbf{k}| \neq\left|\mathbf{k}^{\prime}\right|$. Here, the initial and final states may be designated $|i\rangle=\left|E_{0}^{A} ; n(\mathbf{k}, \lambda) ; 0\left(\mathbf{k}^{\prime}, \lambda^{\prime}\right)\right\rangle$ and $|f\rangle=\left|E_{a}^{A} ; n-1(\mathbf{k}, \lambda) ; 1\left(\mathbf{k}^{\prime}, \lambda^{\prime}\right)\right\rangle$, respectively, the subscript $a$ denoting an excited state of the scatterer in the case of a Stokes transition. Proceeding as before to determine an expression for the quantum amplitude of Raman scattering, the result is expressible as; [10]

$$
M^{f i}=-\left(\frac{\hbar c\left(n k k^{\prime}\right)^{\frac{1}{2}}}{2 \varepsilon_{o} V}\right) e_{i}^{(\lambda)}(\mathbf{k}) \bar{e}_{j}^{\left(\lambda^{\prime}\right)}\left(\mathbf{k}^{\prime}\right) \alpha_{i j}^{a 0(A)}\left(-k^{\prime} ; k\right)
$$

Whilst equation (8) denotes Raman scattering with a Stokes shift, the only difference for the anti-Stokes counterpart is that the superscripts $a$ and 0 (on the $\alpha$ tensor) are interchanged and the emitted wave-vector is understood to correspond to an up-shifted frequency. Through 
the Fermi rule [11], the squared modulus of the quantum amplitudes (6) and (8) delivers the elastic and inelastic scattering rates, respectively.

\subsection{Optical tweezers}

Optical tweezers are instrumental set-ups that enable the physical trapping and manipulation of dielectric micro- and nano-particles. These effects are primarily achieved through an optical phenomenon in which particles are attracted towards a high intensity region (usually at the center) of a focused laser beam. At the heart of this phenomenon is forward Rayleigh scattering, in which $\mathbf{k}=\mathbf{k}^{\prime}$ and $\lambda=\lambda^{\prime}$, and the quantum amplitude is interpreted as a potential energy. Due to the equivalence of the initial and final states for both the molecular and radiation parts of the system, the corresponding matrix element is simply the expectation value of an energy operator. Specifically, given that the initial and final states are given by $|i\rangle=|f\rangle=\left|E_{0}^{A} ; n(\mathbf{k}, \lambda)\right\rangle$, the result is an expression similar in form to equation (6):

$$
M^{f i}=-\left(\frac{n \hbar c k}{2 \varepsilon_{o} V}\right) e_{i}^{(\lambda)}(\mathbf{k}) \bar{e}_{j}^{(\lambda)}(\mathbf{k}) \alpha_{i j}^{00(A)}(-k ; k)-\left(\frac{\hbar c k}{2 \varepsilon_{o} V}\right) e_{i}^{(\lambda)}(\mathbf{k}) \bar{e}_{j}^{(\lambda)}(\mathbf{k}) \sum_{r} \frac{\mu_{i}^{0 r(A)} \mu_{j}^{r 0(A)}}{E_{r 0}+\hbar c k} .
$$

The leading term of (9) represents the potential energy responsible for optical tweezing, as becomes evident on using the irradiance relationship $I=n \hbar c^{2} k / V$. Comparing the classical expression $I=1 / 2 c \mathcal{E}_{0} \mathscr{\mathscr { C }}$, where $\mathscr{E}$ is the amplitude of the electric field, it is evident that this interaction is a quadratic response to the field registered by the polarizability, i.e. an energy of the form $-\alpha\left\langle\mathscr{E}^{2}\right\rangle$. It is because Gaussian beams have their highest intensity at the beam focus that particles are drawn to a local energy minimum. The second term in (9), corresponding to the second in equation (5), accounts for a self-energy correction.

\section{TWO-CENTER SCATTERING}

On addition of a further scattering site $B$, in nanoscale proximity to scatterer $A$, the system becomes electrodynamically much more complex, since the interaction between $A$ and $B$ can occur on essentially the same timescale as the pairwise scattering of the incident radiation, as illustrated by Fig. 3 .

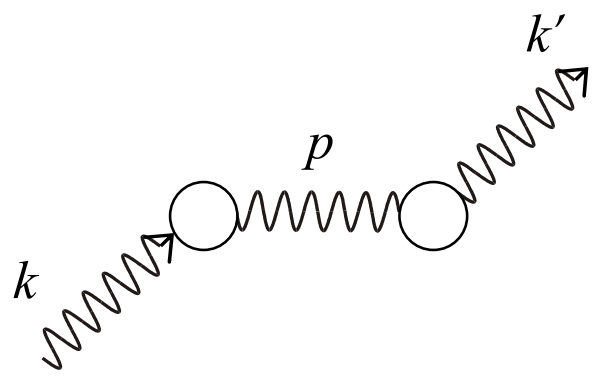

Fig. 3. Representation of bimolecular scattering involving $A$ and $B$ (left and right, respectively). Here, applied radiation is shown incident from below the pair and emergent from above, the horizontal wave denotes pairwise coupling, and $p$ is a coupling photon. 
In the following, we restrict our studies to dynamic optical behavior, not considering contributions due to static fields $[12,13]$ (which only occur in non-centrosymmetric centers where permanent dipole moments are present). Having this in mind, there are four light-matter interactions of relevance: $(a)$ incident light absorption (photon annihilation) at $A$; (b) emergent light emission (photon creation) at $B ;(c)$ a pair coupling event at $A ;(d)$ equivalent to $(c)$ but at $B$. In the QED description, all possible permutations in which these events occur must be considered. As a calculational aid, these permutations are depicted in the state-sequence diagram [14,15] of Fig. 4, within which each of the 24 routes (from the initial on the left to the final state on the right) represent all of the possible state permutations.

In addition, a further case where electromagnetic radiation is absorbed at $B$ and emitted at $A$ must also be included in the calculations since it is physically indistinguishable, i.e. a further 24 permutations have to be accounted for. The corresponding state-sequence diagram, not shown here, is similar to Fig. 4. Bimolecular effects, such as those analogous to Rayleigh and Raman scattering, may all be described by the above explanation. Moreover, bimolecular scattering may be characterized by the simple representation;

$$
A^{0}+B^{0}+\hbar c k \rightarrow A^{a}+B^{b}+\hbar c k^{\prime},
$$

where the superscripts $a$ and $b$ denote excited states for two-centre Raman scattering. For two-centre Rayleigh scattering, $a$ and $b$ equate to the ground state and $k=k^{\prime}$. Following

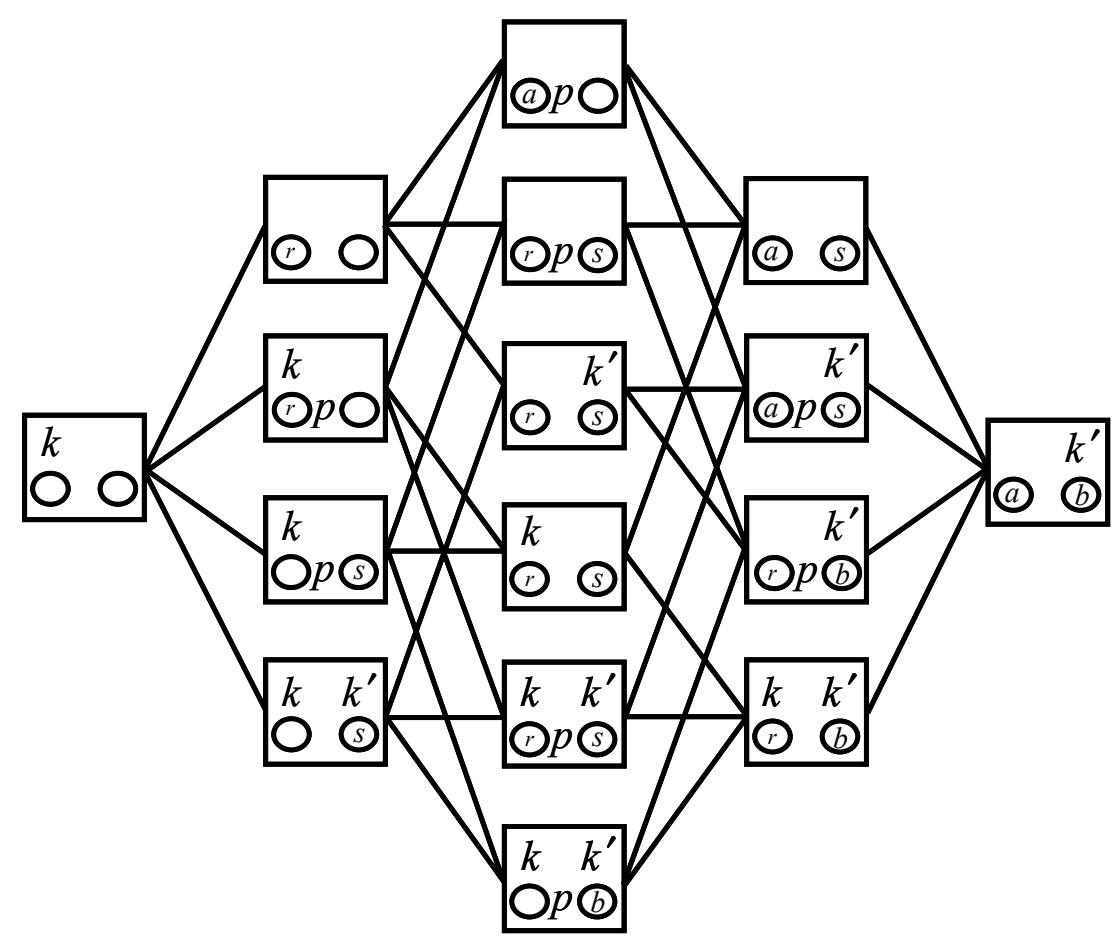

Fig. 4. State-sequence diagram for bimolecular scattering. As Fig. 2, but including (on the right-hand side of each box) a representation of a second scatterer for which $s$ is an intermediate state; $b$ denotes an excited state in Raman scattering, or the ground state for elastic scattering. Here, there are 24 routes from left to right. 
from the previous section, two-centre forward Rayleigh scattering - also known as optical binding - is now to be analyzed. Expressions for the two-center processes analogous to non-forward Rayleigh and Raman scattering are then presented.

\subsection{Optical binding}

Optical binding is an optomechanical effect exhibited by systems of micro- and nanoparticles, suitably irradiated with off-resonance laser light $[12,16]$. Physically distinct from standing-wave and other forms of holographic optical trap, the phenomenon arises as a result of an inter-particle coupling with individual radiation modes, leading to optically induced modifications to Casmir-Polder interactions. These optically induced inter-particle interactions give rise to forces and torques (usually described as optical binding, although the forces are not necessarily attractive in form) and they have been a particular focus in extensive recent investigations, see for example refs [7,17-24] and references therein. The phenomenon has increasingly been advocated as a tool for the optical manipulation and configuration of particles, and many optically induced arrays have been observed experimentally $[25,26]$.

Starting with two centers, $A$ and $B$ in the presence of a laser beam, the 'initial' and 'final' states of the system can be written as;

$$
\begin{aligned}
& |i\rangle=\left|E_{0}^{A}, E_{0}^{B} ; n(\mathbf{k}, \lambda)\right\rangle, \\
& |f\rangle=\left|E_{0}^{A}, E_{0}^{B} ; n(\mathbf{k}, \lambda)\right\rangle,
\end{aligned}
$$

emphasizing that these are identical. Again, the equivalence of the two system state vectors signifies that diagonal elements of the transition matrix are to be derived. The result is therefore to be interpreted as a pair potential energy. Physically, since both the material and radiation parts of the system are identical in $|i\rangle$ and $|f\rangle$, optical binding can thus be interpreted as two-center forward Rayleigh scattering.

As can be seen from expression (11), the particles $A$ and $B$ are generally considered to be in their lowest energy state, i.e. the ground state, and they remain in these states beyond any interaction with the throughput radiation. Four possible mechanisms must be considered. These are: $(a)$ the case where a photon absorption event occurs in centre $A$, and a photon emission event, of the same mode, occurs at centre $B ;(b)$ the second mechanism, which comprises the mirror case where $A$ and $B$ roles are exchanged, $(c)$ and $(d)$ are cases involving the static form of contribution. Since we are considering centrosymmetric (and therefore necessarily non-polar) centers, these last two are not present. Based on the coupling method described in ref. [27], the resulting optically induced energy between the interacting centers emerges as follows:

$$
\begin{aligned}
\Delta E(\mathbf{k}, \mathbf{R})= & \left(\frac{I}{2 \varepsilon_{0} c}\right) e_{i}^{(\lambda)} \bar{e}_{l}^{(\lambda)} \operatorname{Re}\left\{\alpha_{i j}^{A}(-k, k) V_{j k}(k, \mathbf{R}) \alpha_{k l}^{B}(-k, k) \exp (-i \mathbf{k} \cdot \mathbf{R})\right. \\
& \left.+\alpha_{i j}^{B}(-k, k) V_{j k}(k, \mathbf{R}) \alpha_{k l}^{A}(-k, k) \exp (i \mathbf{k} \cdot \mathbf{R})\right\}
\end{aligned}
$$

Here, $V_{j k}(k, \mathbf{R})$ is the retarded electric dipole-dipole coupling tensor. Equation (12) is now used to produce contour plots representing the variation of potential energy, with the positions 
and orientations of each particle relative to each other, and to the throughput radiation (Fig. 5). The results take the form of energy landscapes that exhibit highly detailed topographic features $[22,28]$. The analysis of these features facilitates the determination of possible stability points associated with optical binding, and the identification of other, anisotropic local forces and torques. As such, the pair potential provides a prototypical template for the optical assembly of larger numbers of particles, offering copious possibilities to optically fabricate structures with nanoscale dimensions.
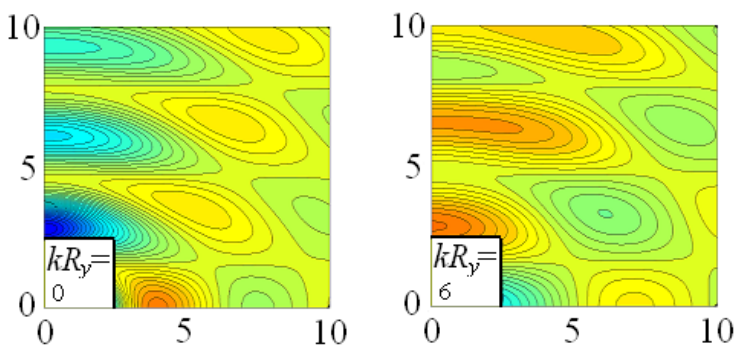

Fig. 5. Illustrative pair potential energy surfaces for optical binding, with the optical field polarization $\boldsymbol{e}$ directed along the $x$-axis (abscissa), wave-vector $\boldsymbol{k}$ along the $z$ axis (ordinate). With one particle placed at the origin, these surfaces are shown as functions of the inter-particle displacement vector, $\boldsymbol{R}=\left(R_{x}, R_{y}, R_{z}\right)$, for different values of $k R_{y}$. Abscissa $k R_{x}$, ordinate $k R_{z}$, each scale unit $2 I \alpha^{2} k^{3} / \varepsilon_{o} c$.

The model described here correlates with experimental work in the identification of stable configurations, as identified by the minima in the potential energy landscapes; at this stage of development it has not yet proved possible to experimentally differentiate between the predictions based on quantum electrodynamical and semiclassical theories. To further develop a link between theory and experiment in the field of optical binding there are certain considerations that must be taken into account. The suitable conditions for the sought effects generally dictate the use of specialized cells or optical traps, each of which may generate additional, partly contributory optical effects - effects that compete with or modify the results of optical binding in many cases.

\subsection{Two-center non-forward Rayleigh scattering}

From the optical binding process which, as we have seen, can be described as two-center forward Rayleigh scattering, it seems natural to consider the non-forward case where the energy states of the two centers still remain unchanged. During this process a photon in mode $(\mathbf{k}, \lambda)$ is annihilated and a photon emerges in a different mode $\left(\mathbf{k}^{\prime}, \lambda^{\prime}\right)$, but with identical energy, i.e. $\hbar c k=\hbar c k^{\prime}$. The initial and final states describing the quantum system are now:

$$
\begin{aligned}
& |i\rangle=\left|E_{0}^{A}, E_{0}^{B} ; n(\mathbf{k}, \lambda), 0\left(\mathbf{k}^{\prime}, \lambda^{\prime}\right)\right\rangle, \\
& |f\rangle=\left|E_{0}^{A}, E_{0}^{B} ; n-1(\mathbf{k}, \lambda), 1\left(\mathbf{k}^{\prime}, \lambda^{\prime}\right)\right\rangle .
\end{aligned}
$$

The process is therefore described in terms of a quantum matrix element, $M^{f i}\left(\mathbf{k}, \mathbf{k}^{\prime}, \mathbf{R}\right)$, given by; 


$$
\begin{aligned}
M^{f i}\left(\mathbf{k}, \mathbf{k}^{\prime}, \mathbf{R}\right)= & \left(\frac{n^{\frac{1}{2}} \hbar c k}{2 \varepsilon_{o} V}\right) \\
& \times\left\{e_{i}^{(\lambda)}(\mathbf{k}) \bar{e}_{l}^{\left(\lambda^{\prime}\right)}\left(\mathbf{k}^{\prime}\right) \alpha_{i j}^{A}(-k, k) V_{j k}(k, \mathbf{R}) \alpha_{k l}^{B}(-k, k) \exp \left(i\left(\mathbf{k} \cdot \mathbf{R}_{A}-\mathbf{k}^{\prime} \cdot \mathbf{R}_{B}\right)\right)\right. \\
& \left.+\bar{e}_{i}^{\left(\lambda^{\prime}\right)}\left(\mathbf{k}^{\prime}\right) e_{l}^{(\lambda)}(\mathbf{k}) \alpha_{i j}^{B}(-k, k) V_{j k}(k, \mathbf{R}) \alpha_{k l}^{A}(-k, k) \exp \left(-i\left(\mathbf{k}^{\prime} \cdot \mathbf{R}_{A}-\mathbf{k} \cdot \mathbf{R}_{B}\right)\right)\right\},
\end{aligned}
$$

where $\mathbf{R}_{\xi}$ denotes the position of centre $\xi$. Through the Fermi rule, this evaluation leads to the determination of an associated scattering rate dependent on the incident irradiance.

However, there are quantum optical issues to consider; the question arises as to whether or not there is an energy associated with (14). In this connection, it is interesting to observe that the QED analysis conveys significant advantages, because it duly registers the change in radiation state. Using a semiclassical approach one would conclude that the initial and final quantum states (matter alone) are identical, whereas the input and output mode distinction in QED signifies an associated change in linear momentum (since a photon with a wave-vector $\mathbf{k}$ carries a linear momentum $\hbar \mathbf{k}$ ). Let us assume that particles $A$ and $B$ are fixed and given by $\mathbf{R}_{A} \equiv 0$ and $\mathbf{R}_{B} \equiv \mathbf{R}$. The dependence on the spatial distribution is evident in equation (14). Since it is an energy rather than a measurement of the emergent radiation that is at issue, theory requires that a summation is performed over the parameters for the unobserved output mode. Duly performing a $4 \pi$ steradian integration over the emission direction for the output radiation mode $\left(\mathbf{k}^{\prime}, \lambda^{\prime}\right)$ results in;

$$
\begin{aligned}
\left\langle M^{f i}\left(\mathbf{k}, \mathbf{k}^{\prime}, \mathbf{R}\right)\right\rangle & =\left(\frac{n^{\frac{1}{2}} \hbar c k}{2 \varepsilon_{o} V}\right)[\frac{i}{3} e_{i}^{(\lambda)}(\mathbf{k}) \alpha_{i j}^{A}(-k, k) V_{j k}(k, \mathbf{R}) \alpha_{k l}^{B}(-k, k) R_{m} \underbrace{\left(\bar{e}_{l}^{\left(\lambda^{\prime}\right)}\left(\mathbf{k}^{\prime}\right) k_{m}^{\prime}\right)}_{=0} \\
& +\underbrace{\left\langle\bar{e}_{i}^{\left(\lambda^{\prime}\right)}\left(\mathbf{k}^{\prime}\right)\right\rangle}_{=0} \alpha_{i j}^{A}(-k, k) V_{j k}(k, \mathbf{R}) \alpha_{k l}^{B}(-k, k) e_{l}^{(\lambda)}(\mathbf{k}) \exp (i \mathbf{k} \cdot \mathbf{R})], \\
\left\langle M^{f i}\left(\mathbf{k}, \mathbf{k}^{\prime}, \mathbf{R}\right)\right\rangle & =0 .
\end{aligned}
$$

Here, to obtain the first line of (15), we used the three-dimensional rotational average identity $\left\langle\mathbf{a} \cdot \mathbf{b}^{\prime} \exp \left(i \mathbf{A} \cdot \mathbf{B}^{\prime}\right)\right\rangle=\frac{i}{3}(\mathbf{a} \cdot \mathbf{A})\left(\mathbf{b}^{\prime} \cdot \mathbf{B}^{\prime}\right)$, where $(\mathbf{a}, \mathbf{A})$ are vectors having a fixed mutual orientation in a particular frame, and $\left(\mathbf{b}^{\prime}, \mathbf{B}^{\prime}\right)$ are mutually fixed vectors in a different reference frame against which the rotational average is carried out. The result given in equation (15) shows that two-center non-forward Rayleigh scattering yields a vanishing optically induced inter-particle energy - and hence no associated pairwise optical forces. The result serves to demonstrate that these energies are produced by the evaluation of expectation values between radiation states that are identical not only in energy, but also in linear momentum.

\subsection{Two-center Raman scattering}

The last process we consider, more briefly, is the two-center counterpart to inelastic scattering [29]. Bimolecular Raman scattering is a process in which the incident radiation undergoes Raman scattering at $A$ and $B$, each of which experiences an uptake (or, conceivably a loss) of energy. Due to the nanoscale proximity of the scattering pair, the incident and emergent light 
again occur essentially instantaneously. Such two-centre Raman scattering has been the subject of relatively few studies, undoubtedly reflecting its experimental difficulties [30]; however it has been suggested that there is a possibility of distinguishing two-center from conventional Raman signals by their anomalously high depolarization ratios [16]. The initial and final states of such process are;

$$
\begin{aligned}
& |i\rangle=\left|E_{0}^{A}, E_{0}^{B} ; n(\mathbf{k}, \lambda), 0\left(\mathbf{k}^{\prime}, \lambda^{\prime}\right)\right\rangle, \\
& |f\rangle=\left|E_{a}^{A}, E_{b}^{B} ; n-1(\mathbf{k}, \lambda), 1\left(\mathbf{k}^{\prime}, \lambda^{\prime}\right)\right\rangle,
\end{aligned}
$$

where $|\mathbf{k}| \neq\left|\mathbf{k}^{\prime}\right|$. In equation (16), both the excitation of the two centers $A$ and $B$ is facilitated by the input radiation mode $(\mathbf{k}, \lambda)$. The output radiation $\left(\mathbf{k}^{\prime}, \lambda^{\prime}\right)$ is such that energy conservation is preserved. The quantum amplitude can readily be obtained;

$$
\begin{aligned}
M^{\mathrm{fi}}\left(\mathbf{k}, \mathbf{k}^{\prime}, \mathbf{R}\right)= & \frac{\hbar c\left(n k k^{\prime}\right)^{\frac{1}{2}}}{2 V \varepsilon_{o}} \\
& \times\left[e_{i}^{(\lambda)}(\mathbf{k}) \alpha_{i j}^{a 0(A)}\left(-k^{\prime}-\frac{E_{b 0}^{B}}{\hbar c} ; k\right) V_{j k}\left(k^{\prime}+\frac{E_{b 0}^{B}}{\hbar c} ; \mathbf{R}\right) \alpha_{k l}^{b 0(\mathrm{~B})}\left(-k^{\prime} ; k-\frac{E_{a 0}^{A}}{\hbar c}\right) \bar{e}_{l}^{\left(\lambda^{\prime}\right)}\left(\mathbf{k}^{\prime}\right) \exp \left(-i \mathbf{k}^{\prime} \cdot \mathbf{R}\right)\right. \\
& \left.+\bar{e}_{i}^{\left(\lambda^{\prime}\right)}\left(\mathbf{k}^{\prime}\right) \alpha_{i j}^{a 0(A)}\left(k-\frac{E_{b 0}^{B}}{\hbar c} ;-k^{\prime}\right) V_{j k}\left(-k+\frac{E_{b 0}^{B}}{\hbar c} ; \mathbf{R}\right) \alpha_{k l}^{b 0(\mathrm{~B})}\left(k ;-k^{\prime}-\frac{E_{a 0}^{A}}{\hbar c}\right) e_{l}^{(\lambda)}(\mathbf{k}) \exp (i \mathbf{k} \cdot \mathbf{R})\right] .
\end{aligned}
$$

The structure of the above and other two-center results invites attention, paving the way for an analysis of multi-center scattering processes - the subject of the next section. Here, attention will focus on optical binding, since this is by far the most experimentally prominent multi-particle optical process. Already, a range of studies has indicated the significant potential that this technique presents for the nanoscale assembly of matter.

\section{MULTI-CENTER OPTICAL BINDING}

The analysis of expression (12), describing optically conferred pair energies, reveals a spatial dependence of the optical energy that leads to richly detailed potential energy landscapes. Based on these results it is possible to extend the analysis of optical binding to multimolecular systems. In general, a determination of the optically induced potential experienced by an assembly of $N$ centers requires the evaluation of equation (12) for $\frac{1}{2} N(N-1)$ pairs obviously making depictions of the system significantly more complex when considering a large number of particles. Beyond the pair approximation for an $N$-center system, further contributions to the optically-induced inter-particle energy may be calculated from first principles based on the state-sequence method. A system involving $N$ centers would entertain $N$ ! mechanisms, each one involving $(2 N)$ ! radiation state-sequences - for example, in the case of a three-center system this would involve consideration of 6 different mechanisms each involving 720 paths, each one cast as a different route through the corresponding statesequence graph. Fortunately, a recently published coupling method [27] offers a straightforward answer to such a problem. This method can deliver a result for a system with an unspecified number of centers. The sought potential energy expression can readily be identified as a sum of all possible contributions of a general form: 


$$
\begin{aligned}
\Delta E^{\left\{\xi_{1}, \ldots \xi_{N}\right\}}= & \frac{n \hbar c k}{2 V \varepsilon_{o}} e_{i_{0}}^{(\lambda)}(\mathbf{k}) \bar{e}_{i_{N}}^{(\lambda)}(\mathbf{k}) \\
& \times \alpha_{i_{i_{1}} i_{1}}^{\left(\xi_{1}\right)}(-k ; k) \prod_{p=1}^{N-1}\left[V_{i_{p} i_{p+1}}\left(k, \mathbf{R}_{\xi_{p} \xi_{p+1}}\right) \alpha_{i_{p+1} i_{p+2}}^{\left(\xi_{p+1}\right)}(-k ; k)\right] \exp \left(-i \mathbf{k} \cdot \mathbf{R}_{\xi_{N} \xi_{1}}\right) .
\end{aligned}
$$

Here, the set of superscript labels $\left\{\xi_{1}, \ldots, \xi_{N}\right\}$ indicates that all possible permutations of the scattering centers $\xi_{i}$ must be taken into account. In particular, for the simplest case of a threecenter system, first studied in ref. [27], we have contributions of the form:

$$
\begin{aligned}
\Delta E^{\{A, B, C\}}= & \frac{n \hbar c k}{2 V \varepsilon_{o}} e_{i_{0}}^{(\lambda)}(\mathbf{k}) \bar{e}_{i_{N}}^{(\lambda)}(\mathbf{k}) \\
& \times \alpha_{i_{i_{0}} i_{1}}^{(A)}(-k ; k) V_{i_{i i_{2}}}\left(k, \mathbf{R}_{B A}\right) \alpha_{i i_{3}}^{(B)}(-k ; k) V_{i_{3,4}}\left(k, \mathbf{R}_{C B}\right) \alpha_{i_{4} i_{s}}^{(C)}(-k ; k) \exp \left(-i \mathbf{k} \cdot \mathbf{R}_{C A}\right) .
\end{aligned}
$$

Accommodating the other 5 permutations of $\{A, B, C\}$, the net result is as follows:

$$
\begin{aligned}
& \Delta E=\frac{n \hbar c k}{2 V \varepsilon_{o}} e_{i_{0}}^{(\lambda)}(\mathbf{k}) \bar{e}_{i_{N}}^{(\lambda)}(\mathbf{k}) \\
& \times\left[\alpha_{i i_{1}}^{(A)}(-k ; k) V_{i i_{2}}\left(k, \mathbf{R}_{B A}\right) \alpha_{i_{2} i_{3}}^{(B)}(-k ; k) V_{i_{3} i_{4}}\left(k, \mathbf{R}_{C B}\right) \alpha_{i_{i, i_{s}}}^{(C)}(-k ; k) \cos \left(\mathbf{k} \cdot \mathbf{R}_{C A}\right)\right. \\
& +\alpha_{i i_{1} i_{1}}^{(A)}(-k ; k) V_{i i_{2},}\left(k, \mathbf{R}_{B A}\right) \alpha_{i i_{3}}^{(C)}(-k ; k) V_{i i_{4} i_{4}}\left(k, \mathbf{R}_{C B}\right) \alpha_{i, i_{j}}^{(B)}(-k ; k) \cos \left(\mathbf{k} \cdot \mathbf{R}_{B A}\right) \\
& \left.+\alpha_{i, i_{1}}^{(B)}(-k ; k) V_{i i_{2}}\left(k, \mathbf{R}_{B A}\right) \alpha_{i_{i j} j_{3}}^{(A)}(-k ; k) V_{i i_{4}}\left(k, \mathbf{R}_{C B}\right) \alpha_{i i_{i j}}^{(C)}(-k ; k) \cos \left(\mathbf{k} \cdot \mathbf{R}_{C B}\right)\right] .
\end{aligned}
$$

The emergent structure gives a strikingly clear indication of the pattern for larger numbers of particles. However it is important to note that, when considering multi-center systems, the optical binding process will be accurately described only when all quantum amplitudes involving different number of centers are considered together, such as those given by equations (12) and (20). This simply reflects the additivity of expectation values corresponding to different orders of perturbation theory.

\section{DISCUSSION}

The above analysis affords a comprehensive and consistent development of theory for multiparticle light scattering. First addressing the QED theory of single-center processes, the derivations of results for Rayleigh and Raman scattering, along with optical tweezer effects, have also served to introduce methods subsequently deployed for the study of more complex, multi-center, forms of scattering. Such processes are to be anticipated whenever throughput radiation can engage with two or more scattering sites in nanoscale proximity. Although the theory for inter-particle interactions such as optical binding and inelastic scattering processes is well established, the connectivity of their mechanisms has not until now received sufficient attention. It has been shown that the various forms of multi-center light scattering can be construed as transitions between different radiation states, the corresponding quantum amplitudes being delivered by the application of standard quantum electrodynamical methods, expedited by a state-sequence development.

As well as offering new insights into the relationship between closely neighboring scattering events, results from the study of two-center Rayleigh scattering have also revealed the origin and consequences of linear momentum conservation. Until now, there has been an 
open question over whether, in the evaluation of a perturbative energy as a diagonal matrix element, it necessary for 'diagonal' to be taken as applying to an exact identity between 'initial' and 'final' states - or whether the restriction should only dictate states of identical energy. In the calculation of the response of a polarizable particle to an oscillating electromagnetic field $\mathscr{E}$, the usual $-\alpha\left\langle\mathscr{C}^{2}\right\rangle$ term in QED language corresponds to the annihilation and stimulated emission of a photon from and 'back into' the throughput light like forward Rayleigh scattering - leading to the known linear dependence on intensity. Evidently, the same logic applies in a multiple scattering case where, following a sequence of matter-radiation interactions, a photon is again delivered back into the throughput beam. Our analysis has shown that no optically induced inter-particle energy - and hence no optical binding - can result if the light emerging from any number of consecutive scattering events is deflected from the direction of initial incidence.

\section{Acknowledgments}

This work is funded by the Engineering and Physical Sciences Research Council, and the Leverhulme Trust. We would like to record our appreciation of Craig Bohren's hugely influential and inspirational work in light scattering; we also thank Rodney Loudon and Peter Milonni for their interest and encouragement concerning our analysis of the quantum optical issues associated with deflected light.

\section{References}

[1] Lord Rayleigh, "On the light from the sky, its polarization and color," in Selected Papers on Scattering in the Atmosphere, C. F. Bohren, Ed., pp. 9-25, SPIE Press, Bellingham, Wash. (1989).

[2] C. F. Bohren and E. Clothiaux, Fundamentals of Atmospheric Radiation, chapter 8, Wiley-VCH, Weinheim, Germany (2006).

[3] C. F. Bohren, "Atmospheric optics," in The Optics Encyclopedia: Basic Foundations and Practical applications, vol. 1, T. G. Brown, K. Creath, H. Kogelnik, M. A. Kriss, J. Schmit, and M. J. Weber, Eds, pp. 53-91, Wiley-VCH, Weinheim, Germany (2004).

[4] C. F. Bohren and D. R. Huffman, Absorption and Scattering of Light by Small Particles, Wiley, New York (1983).

[5] M. M. Burns, J. M. Fournier, and J. A. Golovchenko, "Optical mattercrystallization and binding in intense optical fields," Science 249, 749-754 (1990) [doi:10.1126/science.249.4970.749].

[6] M. Guillon and B. Stout, "Optical trapping and binding in air: Imaging and spectroscopic analysis," Phys. Rev. A 77, $023806 \quad$ (2008) [doi:10.1103/PhysRevA.77.023806].

[7] R. F. Marchington, M. Mazilu, S. Kuriakose, V. Garcés-Chávez, P. J. Reece, T. F. Krauss, M. Gu, and K. Dholakia, "Optical deflection and sorting of microparticles in a near-field optical geometry," Opt. Exp. 16, 3712-3726 (2008) [doi:10.1364/OE.16.003712].

[8] E. A. Power and S. Zienau, "Coulomb gauge in non-relativistic quantum electrodynamics and the shape of spectral lines," Phil. Trans. R. Soc. Lond. A 251, pp. 427-454 (1959) [doi:10.1098/rsta.1959.0008].

[9] E. A. Power, Introductory Quantum Electrodynamics, American Elsevier Pub., New York (1965).

[10] D. P. Craig and T. Thirunamachandran, Molecular Quantum Electrodynamics, chapter 6, Dover, New York (1998). 
[11] L. Mandel and E. Wolf, Optical Coherence and Quantum Optics, p. 871, University Press, Cambridge (1995).

[12] D. S. Bradshaw and D. L. Andrews, "Optically induced forces and torques: Interactions between nanoparticles in a laser beam," Phys. Rev. A 72, 033816 (2005) [doi:10.1103/PhysRevA.72.033816].

[13] A. Salam, "Two alternative derivations of the static contribution to the radiationinduced intermolecular energy shift," Phys. Rev. A 76, 063402 (2007) [doi:10.1103/PhysRevA.76.063402].

[14] R. D. Jenkins, D. L. Andrews, and L. C. Dávila Romero, "A new diagrammatic methodology for non-relativistic quantum electrodynamics," J. Phys. B: At. Mol. Opt. Phys. 35, 445-468 (2002) [doi:10.1088/0953-4075/35/3/301].

[15] A. Salam, Molecular Quantum Electrodynamics: Long-Range Intermolecular Interactions, Wiley, Hoboken, NJ (2010).

[16] D. L. Andrews and P. Allcock, "Polarization analysis of bimolecular excitations mediated by energy-transfer - A common theoretical framework for fluorescence migration and sequential Raman-scattering," Chem. Phys. 198, 35-51 (1995) [doi:10.1016/0301-0104(95)00088-6 ].

[17] T. Thirunamachandran, "Intermolecular interactions in the presence of an intense radiation field," Mol. Phys. 40, 393-399 (1980) [doi:10.1080/00268978000101561].

[18] M. Guillon, O. Moine, and B. Stout, "Longitudinal optical binding of high optical contrast microdroplets in air," Phys. Rev. Lett. 96, 143902 (2006) [doi:10.1103/PhysRevLett.96.143902].

[19] A. Salam, "Intermolecular interactions in a radiation field via the method of induced moments," Phys. Rev. A 73, $013406 \quad$ (2006) [doi:10.1103/PhysRevA.73.013406].

[20] D. L. Andrews, R. G. Crisp, and D. S. Bradshaw, "Optically induced inter-particle forces: From the bonding of dimers to optical electrostriction in molecular solids," J. Phys. B: At. Mol. Opt. Phys. 39, S637-S650 (2006) [doi:0.1088/0953-4075/39/15/S11 ].

[21] T. M Grzegorczyk, B. A. Kemp, and J. A. Kong, "Stable optical trapping based on optical binding forces," Phys. Rev. Lett. 96, 113903 (2006) [doi:10.1103/PhysRevLett.96.113903].

[22] L. C. Dávila Romero, J. Rodríguez, and D. L. Andrews, "Electrodynamic mechanism and array stability in optical binding," Opt. Comm. 281, 865-870 (2007) [doi:10.1016/j.optcom.2007.10.026].

[23] L. C. Dávila Romero and D. L. Andrews, "Nanoscale optics: Interparticle forces," in Structured Light and Its Applications: An Introduction to Phase-Structured Beams and Nanoscale Optical Forces, D. L. Andrews, Ed., pp. 79-105, Academic Press, Burlington, MA (2008).

[24] T. Čižmár, L. C. Dávila Romero, K. Dholakia, and D. L. Andrews, "Multiple optical trapping and binding: New routes to self-assembly," J. Phys. B: At. Mol. Opt. Phys. (in press).

[25] S. K. Mohanty, J. T. Andrews, and P. K Gupta, "Optical binding between dielectric particles," Opt. Exp. 12, 2746-2753 (2004) [doi:10.1364/OPEX.12.002746].

[26] C. D. Mellor, T. A. Fennerty, and C. D. Bain, "Polarization effects in optically bound particle arrays," Opt. Exp. 14, 10079-10088 (2006) [doi:10.1364/OE.14.010079].

[27] L. C. Dávila Romero and D. L. Andrews, "A retarded coupling approach to intermolecular interactions," J. Phys. B: At. Mol. Opt. Phys. 42, 085403 (2009) [doi:10.1088/0953-4075/42/8/085403]. 
[28] J. Rodríguez, L. C. Dávila Romero, and D. L. Andrews, "Optical binding in nanoparticle assembly: Potential energy landscapes," Phys. Rev. A 78, 043805 (2008)[doi:10.1103/PhysRevA.78.043805]

[29] D. L. Andrews and P. Allcock, "Bimolecular photophysics," Chem. Soc. Rev 24, 259-265 (1995) [doi: 10.1039/CS9952400259].

[30] D. L. Andrews and N. P. Blake, "Quantum electrodynamic study of bimolecular scattering effects in Raman-spectroscopy," Phys. Rev. A 41, 2547-2565 (1990) [doi:10.1103/PhysRevA.41.2547].

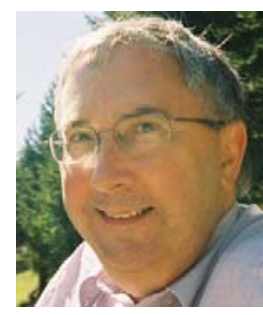

David L. Andrews holds a Chair in Chemical Physics at the University of East Anglia (UEA) in Norwich, U.K. He is a Fellow of the Institute of Physics, the Royal Society of Chemistry, and the International Society for Optical Engineering, SPIE. David's research centers on photophysics, using quantum electrodynamics to develop theory for energy transport and a range of multiphoton and other nonlinear optical phenomena. Current activities focus on nanophotonics, optical binding, and energy harvesting. David has published 250 peer-reviewed papers and ten books.

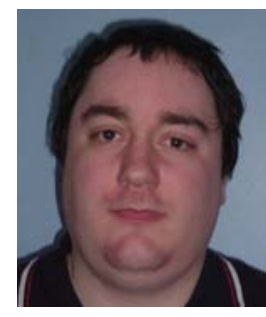

David S. Bradshaw is an Honorary Research Fellow at UEA. He first graduated with a MChem degree from UEA in 2002, including a year at the University of Western Ontario, London, Canada. His $\mathrm{PhD}$ degree in theoretical chemical physics was awarded by UEA in 2006. The author of over 30 peer-reviewed papers, David has also written an encyclopedia article on applied spectroscopy; his current research interests include alloptical switching, resonance energy transfer, and optical binding. He is a member of the Institute of Physics and the Royal Society of Chemistry.

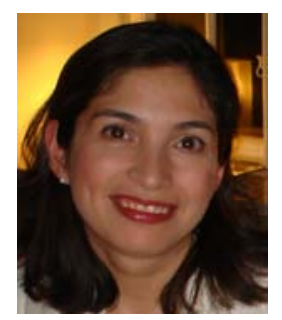

Luciana C. Dávila Romero is a Research Associate at UEA, where her research interests are light-matter interactions based on quantum electrodynamics, particularly optical binding. She obtained her Licentiate degree in Physics at the University of Buenos Aires, Argentina. She has held postdoctoral research appointments at the University of St Andrews and UEA, researching nonlinear optical processes, the angular momentum of light, molecular interactions, quantum optics, etc. Luciana has authored more than 35 peer-reviewed papers, and three co-authored book chapters. 